\title{
Structure and Dynamics of Ribonuclease A During Thermal Unfolding: The Failure of the Zimm Model
}

\author{
Jennifer Fischer ${ }^{\dagger}$, Aurel Radulescu ${ }^{\llbracket}$, Peter Falus ${ }^{\S}$, Dieter Richter ${ }^{\dagger}$, Ralf Biehl ${ }^{* \dagger \ddagger}$, \\ ${ }^{\dagger}$ Jülich Centre for Neutron Science (JCNS-1) \& Institute of Biological Information Processing (IBI-8), \\ Forschungszentrum Jülich GmbH, 52425 Jülich, Germany \\ 'Jülich Centre for Neutron Science JCNS at Heinz Maier-Leibnitz Zentrum (MLZ), \\ Forschungszentrum Jülich, 85748 Garching, Germany \\ ${ }^{8}$ Institute Laue-Langevin (ILL), 71 rue des Martyrs, 38042 Grenoble, Cedex 9, France
}

\section{Supporting information}

\section{DLS}

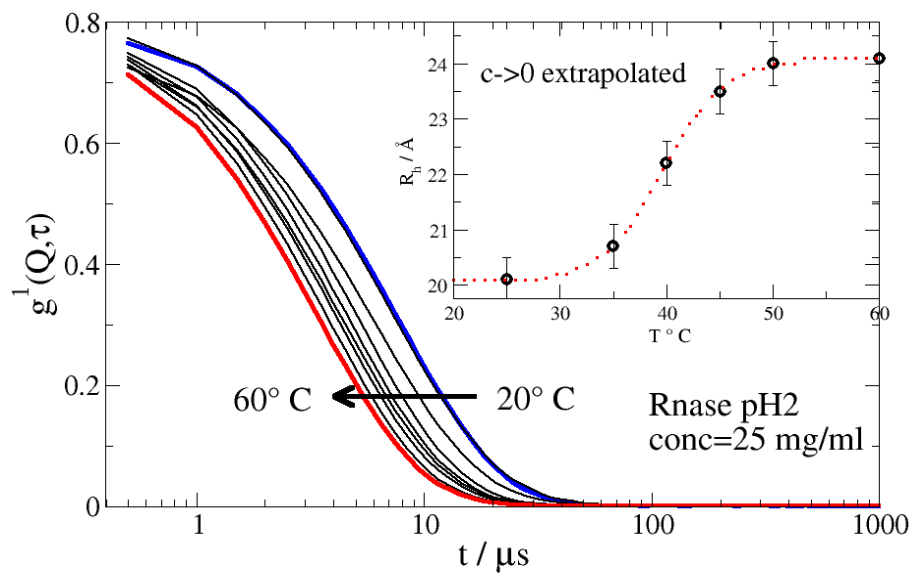

Figure S1 Exemplary DLS correlation functions for Rnase at $\mathrm{pH} 2$ and a concentration of $25 \mathrm{mg} / \mathrm{ml}$. The inset shows the result for the hydrodynamic radius $R_{h}$ from extrapolation to zero concentration. 


\section{Spectroscopy}

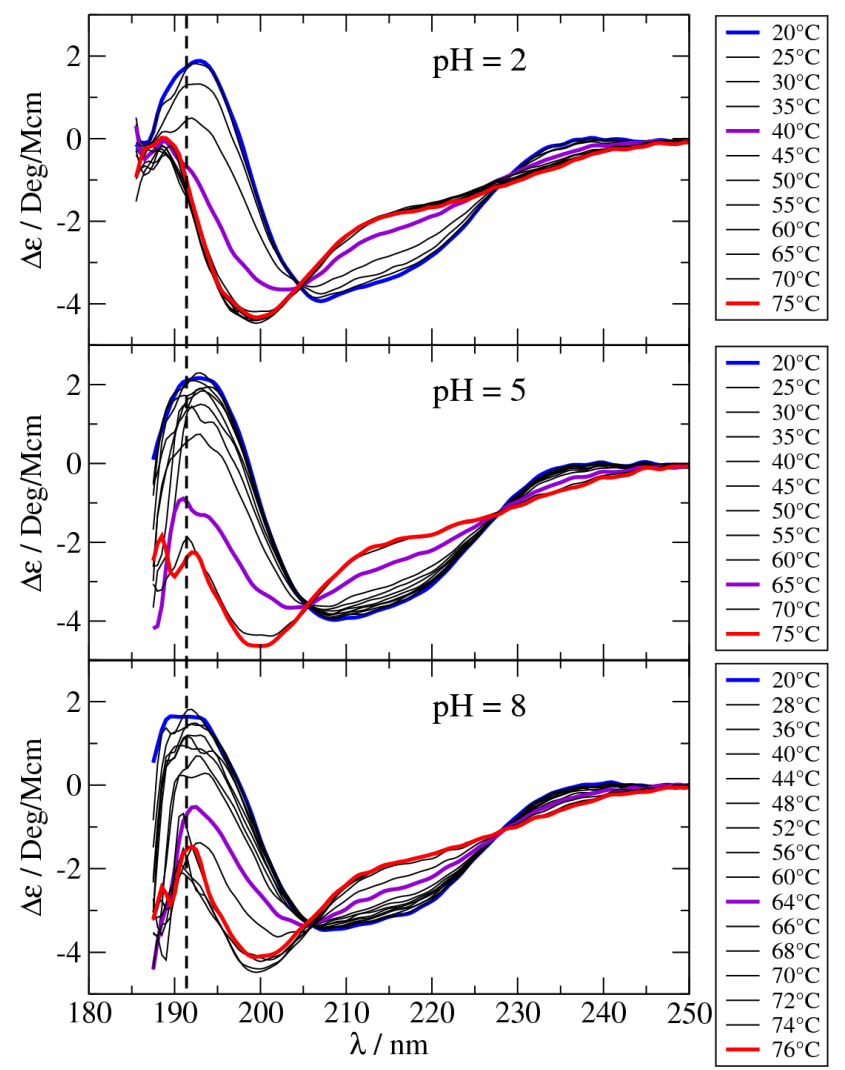

Figure S2 Cd-Spectroscopic Data for Rnase at different $\mathrm{pH}$ for different temperatures as indicated. Data are analyzed using CD-Pro ${ }^{1}$, a program unifying methods as CONTIN, SELCON and CDSSTR, determining the secondary structure content from a fit to the whole spectrum based on reference spectra. Exemplary results are shown in Fig S2.
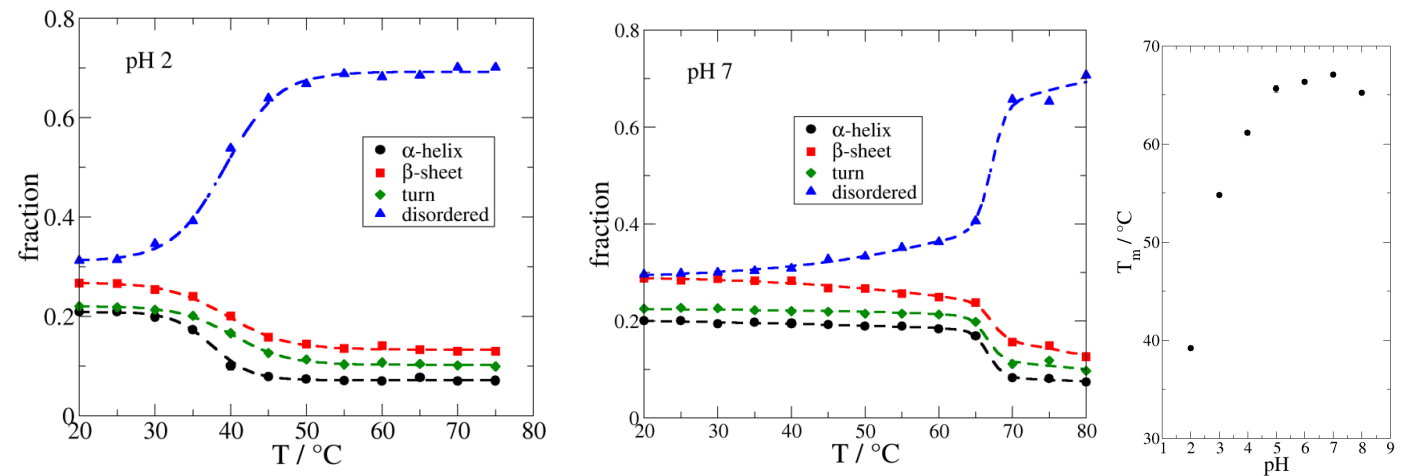

Figure S3 Analysis results of Rnase secondary structure using CD-Pro for $\mathrm{pH} 2$ and $\mathrm{pH} 7$ showing the transitions from mainly $\alpha$-helix and $\beta$-sheet structure to disordered structure. At pH7 a second intermediate transition is observed $\left(T_{\mathrm{mi}}=45^{\circ}\right)$ which is destabilizing $\beta$-sheets. The main transition temperature $\mathrm{T}_{\mathrm{m}}$ is gradually reduced from $67^{\circ}$ at $\mathrm{pH} 7$ to $39^{\circ}$ at $\mathrm{pH} 2$ as shown on the right. Analysis of the spectra as shown in Fig 1 was done by the CD-Pro software. 


\section{DLS and viscosity measurements results}

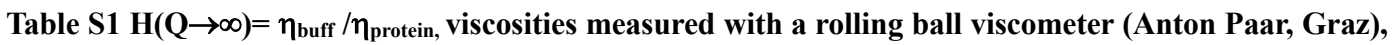

$D_{0}$ single protein diffusion coefficient, $D_{\text {eff }}$ at the used NSE concentration, both extrapolated from a concentration series measured by DLS. Resulting $H(Q)=D_{\text {eff }}(Q) / D_{0} S(Q)$ with $Q \approx 0.0026 \AA^{-1}$ from DLS and $S(Q)$ from SANS.

\begin{tabular}{|c|c|c|c|c|c|c|c|}
\hline $\begin{array}{c}\mathrm{T} \\
{ }^{\circ} \mathrm{C}\end{array}$ & $\begin{array}{c}\eta_{\text {buff }} \\
\mathrm{mPas}\end{array}$ & $\begin{array}{c}\eta_{\text {protein }} \\
\mathrm{mPas}\end{array}$ & $\mathrm{H}(\mathrm{Q} \rightarrow \infty)$ & $\begin{array}{c}\mathrm{D}_{0} \\
\AA^{2} / \mathrm{ns}\end{array}$ & $\begin{array}{c}\mathrm{D}_{\text {eff }} \\
\AA^{2} / \mathrm{ns}\end{array}$ & $\mathrm{S}(\mathrm{Q} \approx 0)$ & $\mathrm{H}(\mathrm{Q} \approx 0)$ \\
\hline 20 & 1.285 & 1.640 & 0.783 & 9.3 & 9.8 & 0.508 & 0.535 \\
\hline 35 & 0.892 & 1.158 & 0.770 & 12.0 & 14.8 & 0.484 & 0.596 \\
\hline 40 & 0.804 & 1.076 & 0.747 & 12.9 & 16.3 & 0.449 & 0.564 \\
\hline 45 & 0.730 & 1.027 & 0.711 & 14.3 & 17.4 & 0.432 & 0.522 \\
\hline 50 & 0.667 & 0.976 & 0.683 & 15.0 & 18.7 & 0.445 & 0.554 \\
\hline 60 & 0.565 & 0.837 & 0.675 & 17.9 & 21.9 & 0.514 & 0.627 \\
\hline
\end{tabular}

\section{Coarse Grain Simulation}

To obtain structures of unfolded RNase $\mathrm{A}$ that describe the measured form factor $\mathrm{P}(\mathrm{Q})$ at $\mathrm{T}=$ $60{ }^{\circ} \mathrm{C}$ coarse-grained Brownian dynamic simulations were performed. For this purpose the program suite SIMUFLEX was used ${ }^{2}$. Following parameters are used according to Amoros et al. who simulated disordered proteins within a simple coarse-grained model ${ }^{3}$.

For the simulation, each amino acid of RNase is represented by one bead with a hard sphere radius of $\mathrm{R}_{\mathrm{HS}}=2 \AA$ and a hydrodynamic radius of $\mathrm{R}_{\mathrm{h}}=6.1 \AA$. The beads are connected by hard springs:

$$
V^{\operatorname{Conn}}(l)=\frac{1}{2} H\left(l-l_{e}\right)^{2}
$$

with an equilibrium length $\mathrm{l}_{\mathrm{e}} \approx 3.8 \AA$ being the $\mathrm{C}_{\alpha}-\mathrm{C}_{\alpha}$ distance from the crystal structure that is used as the initial conformation of the simulation. The force constant $\mathrm{H}$ can be calculated using $l_{\mathrm{e}}$ :

$$
H=\frac{f k_{B} T}{l_{e}^{2}} \approx 2700 \frac{e r g}{\mathrm{~cm}}
$$

with $\mathrm{T}=283.15 \mathrm{~K}=10{ }^{\circ} \mathrm{C}$ and $\mathrm{f}=100$ that gives rms fluctuations of $10 \%$ around the equilibrium position $\mathrm{l}_{\mathrm{e}}$. The potential for the bending angles of neighboring $\mathrm{C}_{\alpha}$ atoms is given by:

$$
V^{a n g}(\alpha)=\frac{1}{2} Q\left(\alpha-\alpha_{0}\right)
$$

with $\alpha_{0}=112^{\circ}$ and $\mathrm{Q}=3.5 \mathrm{kcal}$ typical for free angles in unfolded proteins. As the torsion angles of a virtual chain are shown to spread over the whole range from $0^{\circ}$ to $360^{\circ}$, torsion angles are not included in the simulation ${ }^{4}$. Excluded volume interactions are approximated using a soft exponential repulsion:

$$
V^{E V, p a i r s}(r)=A e^{-b r}
$$

with $\mathrm{A}=1.39 \times 10^{-8} \mathrm{erg}$ and $\mathrm{b}=2.875 \times 10^{10} \AA^{-1}$ that are tuned to have the same slope as a Lennard-Jones interaction at $2 \mathrm{R}_{\mathrm{HS}}$ used by Amoros ${ }^{3}$.

The simulations were run using Iniesta-Garcia de la Torre algorithm with a modified Oseen 
tensor and using Choleski decomposition. For further details of the algorithm see ref ${ }^{2}$. The total simulation time after a short equilibration run of $10 \mathrm{~ns}$ was $1 \mu$ s with 5000 conformations in $2 \mathrm{~ns}$ steps saved in the analyzed trajectory. The duration of each simulation step was $0.01 \mathrm{~ns}$. We simulated a Rnase chain without disulfide bonds as free chain and one including the 4 disulfide bonds between cysteines 26-84, 58-110, 40-95, 65-72 as hard springs with an equilibrium bond length as found in the crystal structure. The simulation trajectories are stable within short times and show fluctuations around an average radius of gyration of $20.1 \AA$ for the disulfide containing trajectory and $30 \AA$ for the free chain.

Figure S4 shows the scattering curves calculated from the dummy model. For this each bead was identified as the corresponding amino acid and the pre-calculated amino acid formfactor amplitude $\mathrm{F}_{\mathrm{a}}(\mathrm{Q})$ was used for the corresponding bead to calculate realistic SAXS/SANS formfactors including the respective contrast. For the bead model a hydration layer was not included as the resulting shape of the formfactor didn't change.

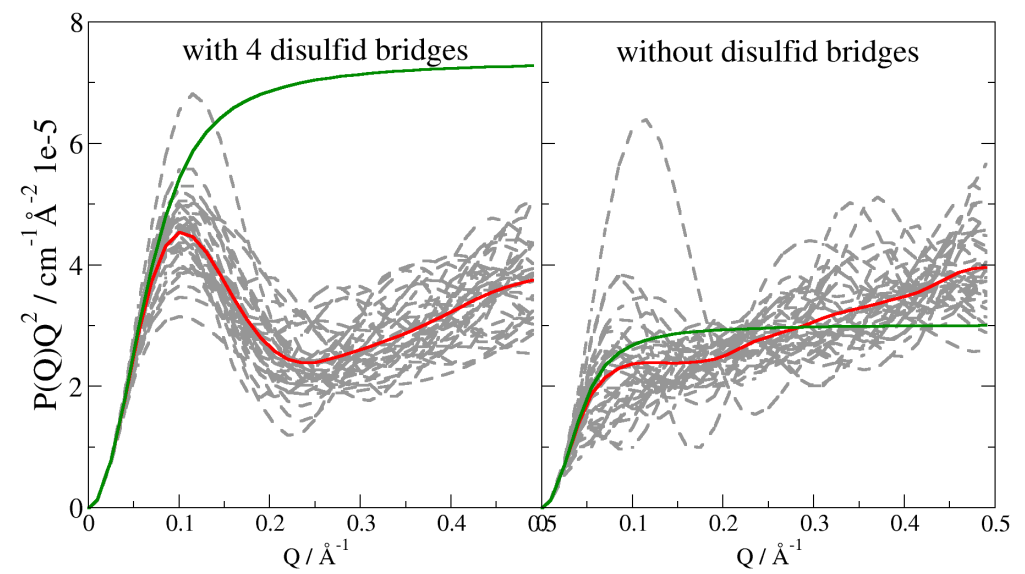

Figure S4: Kratky plot of the SANS formfactor from coarse grained simulation (124 c-alpha atoms) using the program Simuflex ${ }^{2}$. On the left the 4 disulfide bridges found in Rnase are included while on the right the chain contains no disulfide bridges and represents a c-alpha atom Gaussian chain. Grey lines represent individual configurations. The red lines are the time averaged formfactors. Green lines represent the expectation of a Gaussian chain (Debye function) with the same radius of gyration. The increase of the simulated formfactors above $0.2 \mathrm{~A}^{-1}$ corresponds to the observation of linear chain segments on the corresponding length scale and deviate correspondingly from the expectation of a Gaussian chain.

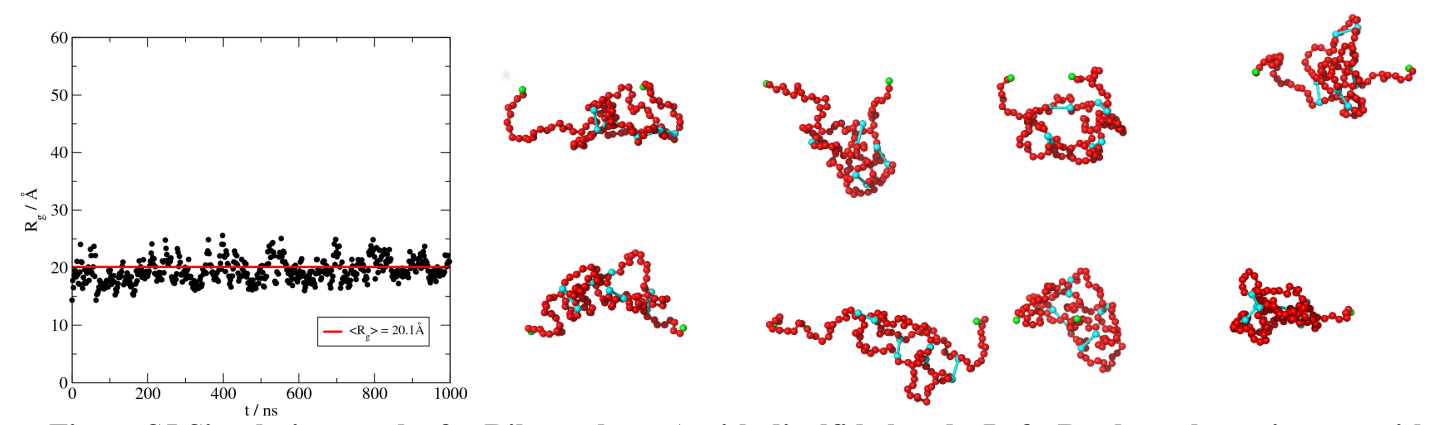

Figure S5 Simulation results for Ribonuclease A with disulfide bonds. Left: $R_{g}$ along the trajectory with an average of 20.1 Å. Right: Chosen 8 configurations that in average describe SANS data best. 


\section{NSE Spectra}

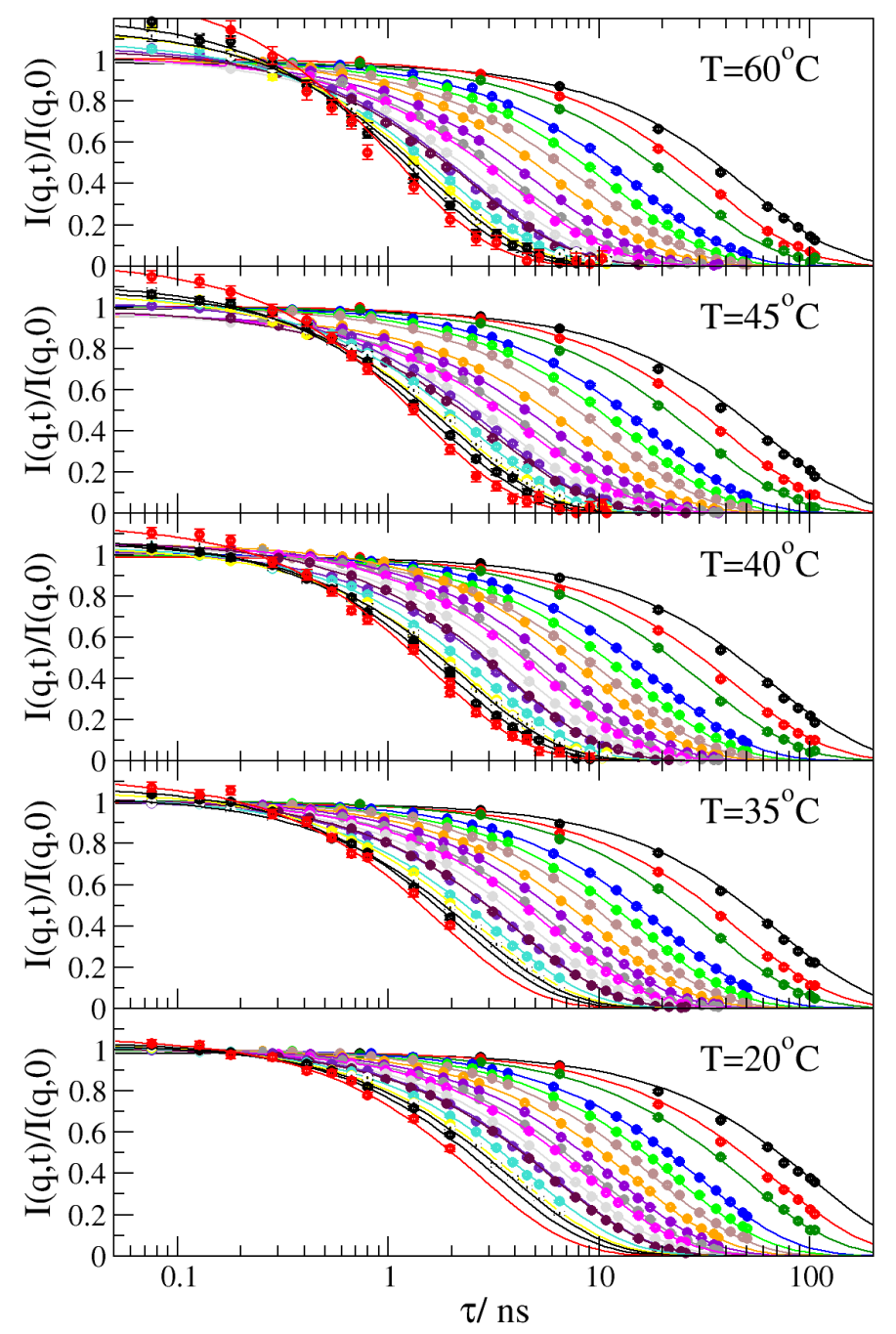

Figure S6 Intermediate scattering function as measured by NSE for Ribonuclease at temperatures as indicated. Lines correspond to the respective model fits as described in the text. $Q$ values from $0.032 \AA^{-}$ ${ }^{1}$ (black, right) to $0.22 \AA^{-1}$ (green, left) as also shown in Fig. 6 main text. Errors are of symbol size or smaller. Incoherent solvent scattering from $\mathrm{D}_{2} \mathrm{O}$ and residual $\mathrm{H}_{2} \mathrm{O}(0.01 \%)$ shows on times $\mathrm{t}<0.5 \mathrm{~ns}$ an increase in background $I(Q, t)$ due to solvent self-diffusion $\left(D_{2} O\right.$ diffusion constant $\left.\approx 170 \AA^{2} / n s\right)$ which is relaxed after $\approx 0.5 \mathrm{~ns}$. The increase is typical for incoherent dynamics opposite to the decay of the sample coherent signal. For same temperature the background correction removes this (see $\mathrm{T}=20^{\circ} \mathrm{C}$ ). For increased temperature $\left(\mathrm{T}>\mathbf{2 0}{ }^{\circ} \mathrm{C}\right)$ water diffusion gets faster and leads to an overshoot at short times for $\mathrm{T}>\mathbf{2 0}{ }^{\circ} \mathrm{C}$. For times $\mathbf{t}>0.5 \mathrm{~ns}$ the effect leads to an amplitude $>1$ which can be accounted for in a model using a respective larger amplitude. See Biehl et al. for details of the background correction ${ }^{5}$. 


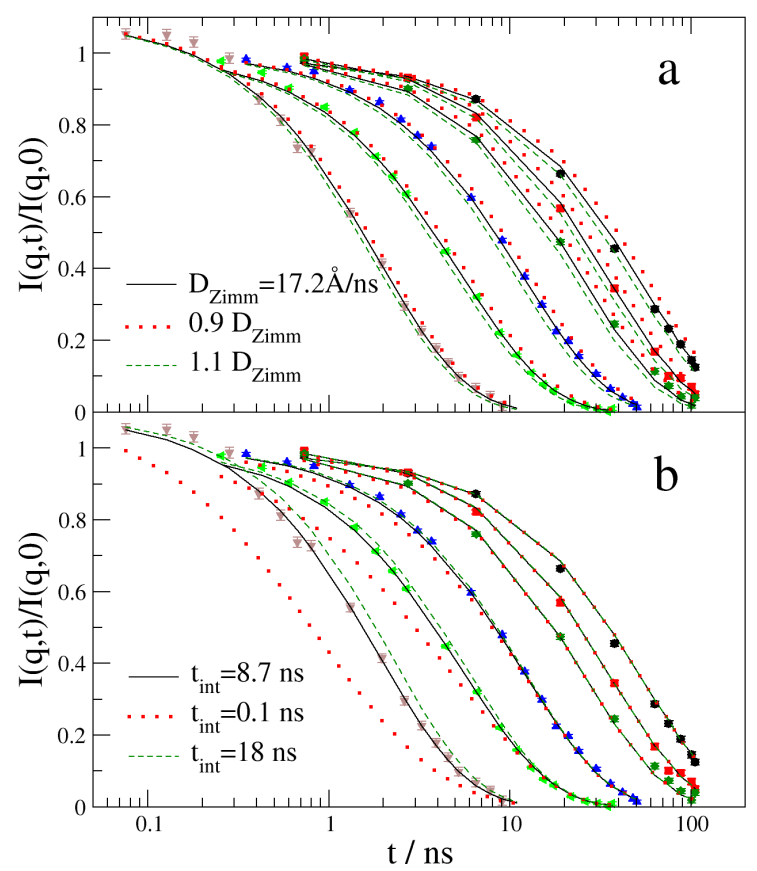

Figure $S 7$ Effect of parameters $D_{\text {Zimm }}$ and $\tau_{\text {int }}$ on the fit result for $T=60^{\circ} \mathrm{C}$ with $D_{\text {Zimm }}=17.2 \AA^{2} / \mathrm{ns}$ and $\tau_{\text {int }}=8.7 \mathrm{~ns}$ for selected $Q$ values $\left(0.32,0.40,0.48,0.75,1.1,1.69 \AA^{-1}\right)$. a: Variation of $D_{\text {Zimm }}$ for $\pm 10 \%$. A parallel shift of all data is observed. As the low $Q$ are not sensitive for $\tau_{\text {int }}$ these are sensitive for the diffusion coefficient. b: Variation between negligible and doubled $\tau_{\text {int }}$ compared to the fit result. The low $Q$ relaxation is uninfluenced while the high $Q$ is strongly influenced by $\tau_{\text {int. }}$. The asymmetric looking effect is related to the used $x-\log$ scale.

\section{References}

(1) Sreerama, N.; Woody, R. W. Estimation of Protein Secondary Structure from Circular Dichroism Spectra: Comparison of CONTIN, SELCON, and CDSSTR Methods with an Expanded Reference Set. Anal. Biochem. 2000, 287 (2), 252260. https://doi.org/10.1006/abio.2000.4880.

(2) de la Torre, J. G.; Cifre, J. G. H.; Ortega, Á.; Schmidt, R. R.; Fernandez, M. X.; Sánchez, H. E. P.; Pamies, R. SIMUFLEX : Algorithms and Tools for Simulation of the Conformation and Dynamics of Flexible Molecules and Nanoparticles in Dilute Solution. J. Chem. Theory Comput. 2009, 5 (10), 2606-2618. https://doi.org/10.1021/ct900269n.

(3) Amorós, D.; Ortega, A.; De La Torre, J. G. Prediction of Hydrodynamic and Other Solution Properties of Partially Disordered Proteins with a Simple, Coarse-Grained Model. J. Chem. Theory Comput. 2013, 9 (3), 1678-1685. https://doi.org/10.1021/ct300948u.

(4) Kleywegt, G. J. Validation of Protein Models from $\mathrm{C}(\alpha)$ Coordinates Alone. $J$. Mol. Biol. 1997, 273 (2), 371-376. https://doi.org/10.1006/jmbi.1997.1309.

(5) Biehl, R.; Monkenbusch, M.; Richter, D. Exploring Internal Protein Dynamics by Neutron Spin Echo Spectroscopy. Soft Matter 2011, 7 (4), 1299. https://doi.org/10.1039/c0sm00683a. 